\title{
Stabilizing Function of the Musculoskeletal System for Periodic Motion
}

\section{AUTHOR(S):}

Sugimoto, Yasuhiro; Aoi, Shinya; Ogihara, Naomichi; Tsuchiya, Kazuo

\section{CITATION:}

Sugimoto, Yasuhiro ...[et al]. Stabilizing Function of the Musculoskeletal System for Periodic Motion. ADVANCED ROBOTICS 2009, 23(5): 521-534

\section{ISSUE DATE:}

2009

URL:

http://hdl.handle.net/2433/109954

\section{RIGHT:}

(C) Koninklijke Brill NV, Leiden and The Robotics Society of Japan, 2009 


\title{
Stabilizing Function of the Musculoskeletal System for Periodic Motion
}

\author{
Yasuhiro Sugimoto $^{\mathrm{a}, *}$, Shinya Aoi ${ }^{\mathrm{b}}$, Naomichi Ogihara ${ }^{\mathrm{c}}$ and Kazuo Tsuchiya ${ }^{\mathrm{d}}$ \\ ${ }^{a}$ Department of Mechanical Engineering, Kobe University, 1-1 Rokkoudai-cho, Nada, \\ Kobe 657-8501, Japan \\ ${ }^{\mathrm{b}}$ Department of Aeronautics and Astronautics, Graduate School of Engineering, Kyoto University, \\ Yoshida-honmachi, Sakyo, Kyoto 606-8501, Japan \\ ${ }^{\mathrm{c}}$ Laboratory of Physical Anthropology, Graduate School of Science, Kyoto University, \\ Kitashirakawa-oiwakecho, Sakyo, Kyoto 606-8502, Japan \\ ${ }^{d}$ Department of Energy and Mechanical Engineering, Faculty of Science and Engineering, \\ Doshisha University, YM420, 1-3 Tatara Miyakodani, Kyotanabe City, Kyoto 610-0321, Japan
}

Received 7 November 2008; accepted 24 December 2008

\begin{abstract}
Animals generate various motions by cooperatively manipulating their complicated and redundant musculoskeletal systems controlled by the nervous system. To reveal the mechanism with which a steady movement is generated, much research has been performed that shows that musculoskeletal properties themselves can stabilze motion without a sensory feedback system if the muscle architecture and the parallel elastic elements within a muscle are tuned appropriately. In this paper, we show that the condition of stability is greatly eased by limiting the targeted movement to the periodic motion. The force-velocity relationship, which is one of the most famous properties of muscle, plays a decisive role in realizing the self-stabilizing ability of the musculoskeletal system.
\end{abstract}

(C) Koninklijke Brill NV, Leiden and The Robotics Society of Japan, 2009

Keywords

Self-stability, musculoskeltal system, preflex, periodic motion

\section{Introduction}

Animals, including humans, achieve various movements by skillfully and cooperatively moving their redundant and complex musculoskeletal system. These movements are undoubtedly ruled by nervous systems in the brain and/or the spinal cord. To clarify the mechanism of generation and the control of movement with the ner-

\footnotetext{
* To whom correspondence should be addressed. E-mail: yas@ osukalab.mech.kobe-u.ac.jp 
vous system, recently research on 'system biomechanics' has started in which the musculoskeletal system model and the neural network model in the spinal cord and/or the brain are integrated.

In this system biomechanics research, Loeb coined the term 'preflex' [1], which mean the zero-delay, intrinsic response of the neuromusculoskeletal system to a perturbation. In the feedback loop of neural networks, a time delay caused by neurotransmission or information processing, etc., inevitably exists. Therefore, using only the feedback control of neural networks, it might be very difficult to realize stable motions, especially to realize fast movements. Although many researchers have tried to clarify how a feedforward control system based on an internal model realized in the nervous system has been achieved, some researchers have also tried to reveal how the 'preflex' properties of musculoskeletal systems contribute to the stability of movements [2-5]. By using a very simple model intended for human bending and stretching exercises, Wagner and Blickhan derived a condition in which the movement driven by the muscle stabilized and showed that the musculoskeletal system had 'self-stability' to some extent, i.e., if the structure of a musculoskeletal system and a muscle viscoelasticity element are appropriately adjusted, the movement of the musculoskeletal system can be stabilized without feedback from the nervous system [4].

There are also various periodic motions such as walking, swimming, flapping and breathing in the movements of animals. As these movements are basically steady motions, it is desirable if the musculoskeletal system has good properties for periodic motions from the viewpoint of a control theory. It is common knowledge that a decerebrate cat can walk on a treadmill [6]. This means that a periodic motion can be generated merely by a relatively low level of the neuro-musculoskeletal system. From the above facts, the self-stability of the musculoskeletal system is considered more important for periodic movements than for other voluntary movements.

In this paper, by limiting the target motions to periodic motions and using the Floquet theorem, we derive an eased stability condition compared to previous work. The derived condition is considered from the viewpoint of physiology.

This paper consists of five sections. After the Introduction in this first section, Section 2 describes the model of the musculoskeletal system. In Section 3, the stability criteria for periodic motions are derived. In Section 4, the interesting property in the derived condition for stability is described and how stability criteria changes when the musculoskeletal system changes is discussed. Finally, Section 5 presents the conclusion.

\section{Model of the Musculoskeletal System}

\subsection{Skeletal Model}

The linkage model that we consider is shown in Fig. 1. It is composed of two massless segments (thigh and shank), a point mass $m$ that represents the body and a muscle (knee extensors). In this paper, we examine the vertical oscillations of this 


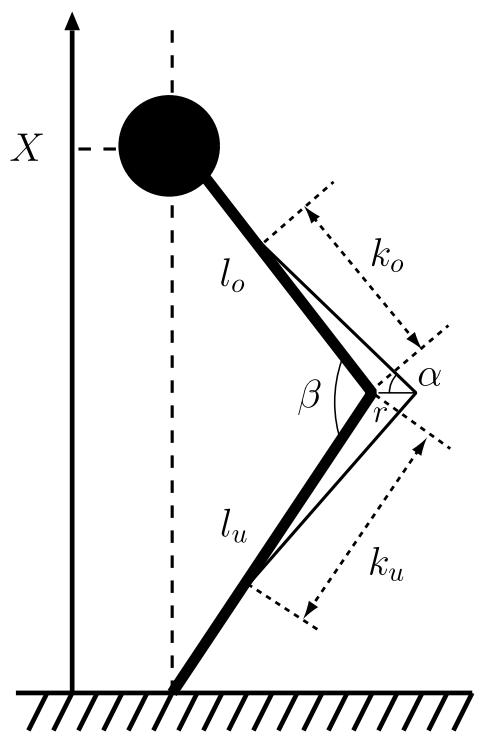

Figure 1. Geometric model of the knee joint. $l_{\mathrm{o}}, l_{\mathrm{u}}$ : length of the thigh and the shank; $k_{\mathrm{o}}, k_{\mathrm{u}}$ : length of the extensor from attachment to the middle of the patella to its insertion; $r$ : distance between the center of rotation and mid-patella to its insertion; $m$ : body mass; $X$ : vertical displacement of the location of the hip.

model that are generated by bending the knees. It is considered that this motion simulates the human knee-bending motion. For simplicity, we assume that the hip does not move horizontally, i.e., it only makes a vertical one-dimensional movement.

Let the position (vertical displacement) of the hip be $X$, its velocity be $V=\dot{X}$, the length of the muscles be $l_{\mathrm{m}}$ and the shortening velocity of muscle be $v_{\mathrm{m}}=-\dot{l}_{\mathrm{m}}$. The moment arm of the knee joint $r$ is assumed to be constant.

The geometric transformation between ground reaction force $F(X, V)$ and muscle force $f_{\mathrm{m}}(t, X, V)$ for vertical movements can be described by function $G(X)$ :

$$
\begin{gathered}
F(X, V)=G(X) f_{\mathrm{m}}(t, X, V) \\
G(X)=\frac{r \sin \alpha}{l_{\mathrm{o}} l_{\mathrm{u}} \sin \beta} X,
\end{gathered}
$$

where:

$$
\begin{gathered}
\beta=2 \alpha+\arcsin \left(\frac{r}{k_{\mathrm{o}}} \sin \alpha\right)+\arcsin \left(\frac{r}{k_{\mathrm{u}}} \sin \alpha\right) \\
X=\sqrt{l_{\mathrm{o}}^{2}+l_{\mathrm{u}}^{2}-2 l_{\mathrm{u}} l_{\mathrm{o}} \cos \beta} .
\end{gathered}
$$

These formulations can be derived with the geometric restraints and the balance of moments. Refer to Refs $[4,7]$ for the detailed devivation of these equations.

Note that inequality $G(X)>0$ always exists for the range of the movement of the knee joint $(0<\alpha<\pi$ and $0<\beta<\pi)$. 
With the same function $G(X)$, the relationship between $V$ and $v_{\mathrm{m}}$ can be written as:

$$
V=\frac{1}{G(X)} v_{\mathrm{m}}
$$

\subsection{Muscle Model}

The important factors for the force development of a muscle are contraction velocity $v_{\mathrm{m}}$ and activation function $E$. In this paper, as a force developing model, we adopt the following model described by the product of the simplest muscle model (the Hill-simple [8]) and activation function $E(0 \leqslant E(t) \leqslant 1)$ :

$$
f_{\mathrm{m}}(t, X, V)=E(t) f_{\mathrm{H}}(X, V) .
$$

For concentric contrations, the relationship between force and velocity of muscle $f_{\mathrm{H}}(X, V)$ is:

$$
f_{\mathrm{H}}(X, V)=\frac{c}{v_{\mathrm{m}}(X, V)+b}-a \quad \forall v_{\mathrm{m}} \geqslant 0
$$

For eccentric contrations:

$$
f_{\mathrm{H}}(X, V)=\frac{C}{v_{\mathrm{m}}(X, V)-B}+A \quad \forall v_{\mathrm{m}}<0 .
$$

Both parameters $a, b, c$ and $A, B, C$ in (5) and (6) are determined experimentally $[7,9]$. Thus, accurately, model $f_{\mathrm{H}}(X, V)$ becomes a different model based on the sign of $v_{\mathrm{m}}$. However, for the following discussion, it is assumed to be continuous close to $v_{\mathrm{m}}=0$ (Fig. 2).

There is one very important property of $f_{\mathrm{H}}(X, V)$. From (3), (5) and (6), and the fact that $c$ and $C$ are always positive, $f_{\mathrm{H}}(X, V)$ is monotonically decreasing, i.e.:

$$
\frac{\partial f_{\mathrm{H}}(X, V)}{\partial V}<0,
$$

is always satisfied. This fact should be remembered because it plays a crucial role in the following discussion.

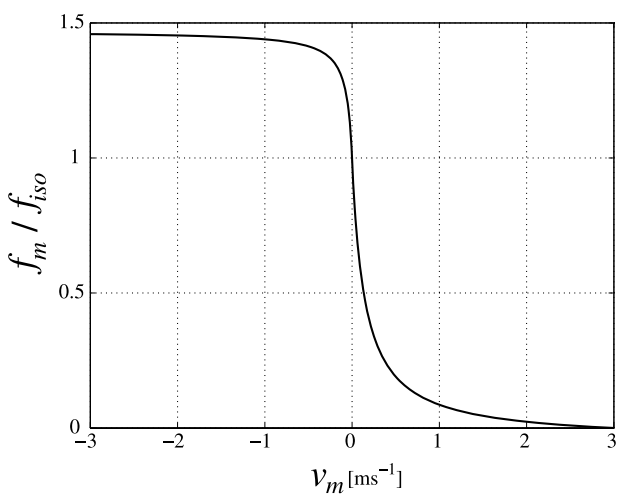

Figure 2. Example of Hill-type force-velocity relation. $f_{\text {iso }}$ is the isometric force of muscle. 


\subsection{Dynamic Equation}

With the geometric function $G(X)$ and the muscle model $f_{\mathrm{m}}(t, X, V)$, the dynamical equation of the system can be described as:

$$
\frac{1}{\mathrm{~d} t}\left(\begin{array}{l}
X \\
V
\end{array}\right)=\left(\begin{array}{c}
V \\
\frac{1}{m} G(X) f_{\mathrm{m}}(t, X, V)-g
\end{array}\right)\left(\begin{array}{c}
V \\
M(t, X, V)
\end{array}\right),
$$

where $g$ is the gravitational acceleration and $M(t, X, V)=1 / m G(X) f_{\mathrm{m}}(t, X$, V) $-g$.

\section{Stability Criteria for Periodic Motion}

In previous work [4], a linearized system of (8):

$$
\frac{1}{\mathrm{~d} t}\left(\begin{array}{l}
X \\
V
\end{array}\right)=\left(\begin{array}{ll}
\frac{\partial V}{\partial X} & \frac{\partial V}{\partial V} \\
\frac{\partial M}{\partial X} & \frac{\partial M}{\partial V}
\end{array}\right)\left(\begin{array}{l}
X \\
V
\end{array}\right)=\left(\begin{array}{cc}
0 & 1 \\
a_{2} & a_{1}
\end{array}\right)\left(\begin{array}{l}
X \\
V
\end{array}\right),
$$

was derived first and the eigenvalues of the Jacobian were derived. Then, from the condition that all these eigenvalues become negative, the stability condition of the system was derived:

$$
a_{1}<0 \quad a_{2}<0 .
$$

With this condition, various discussions were carried out. Here,

$$
\begin{aligned}
& a_{1}=\frac{\partial}{\partial V}\left(\frac{1}{m} G(X) f_{\mathrm{m}}(t, X, V)\right) \\
& a_{2}=\frac{\partial}{\partial X}\left(\frac{1}{m} G(X) f_{\mathrm{m}}(t, X, V)\right),
\end{aligned}
$$

and the activation function of muscle $E(t)$ was assumed to be sufficient to generate a prescribed movement and no delay was assumed in the signal transmission.

In this paper, by limiting target motions $\tilde{X}$ to periodic motions, we try to ease the stability condition (10) with the Floquet theorem.

The eased condition of stability is given by the following theorem.

Theorem 1. System (9) becomes stable for periodic motions if and only if:

$$
\int_{0}^{T} a_{1}(t) \mathrm{d} t<0 \Longleftrightarrow \int_{0}^{T}\left(\frac{\partial}{\partial V}\left(\frac{1}{m} G(X) f_{\mathrm{m}}(t, X, V)\right)\right) \mathrm{d} t<0 .
$$

Proof. Let the one of the periodic solution of (9) whose period is $T$ be:

$$
\Theta_{1}=(\theta(t), \dot{\theta}(t))^{T},
$$

where $\theta(t)=\theta(t+T)$ and $\Theta_{1}$ is not identically zero, i.e., $\theta(t) \not \equiv 0$ and $\dot{\theta}(t) \not \equiv 0$, and $\theta(t)>0$ can be assumed from the fact $X>0$. 
With $u(t)$ and $v(t)$, the another solution $\Theta_{2}$, which is linealy independent of $\Theta_{1}$, can be written as:

$$
\Theta_{2}=(u(t) \theta, v(t) \dot{\theta})^{T} .
$$

From the fact that $\Theta_{2}$ is the solution of (9):

$$
\begin{gathered}
\dot{u} \theta+u \dot{\theta}=v \dot{\theta} \\
\dot{v} \dot{\theta}+v \ddot{\theta}=a_{2} u \theta+a_{1} v \dot{\theta},
\end{gathered}
$$

can be derived. As $\Theta_{1}$ is also the solution of (9):

$$
\ddot{\theta}=a_{2} \theta+a_{1} \dot{\theta},
$$

can be derived. By eliminating $v$ from (15) and (16):

$$
2 \dot{u} \dot{\theta}+\theta \ddot{u}+u \ddot{\theta}=a_{2} u \theta+a_{1}(u \dot{\theta}+\theta \dot{u}) .
$$

By substituting (17) into (18):

$$
\ddot{u}=a_{1} \dot{u}-2 \dot{u} \frac{\dot{\theta}}{\theta},
$$

can be derived.

From (19), $u(t)$ and $\dot{u}(t)$ can be described as follows:

$$
\begin{aligned}
& \dot{u}(t)=C_{2} \mathrm{e}^{\int_{0}^{t}\left(a_{1}(s)-2 \dot{\theta}(s) / \theta(s)\right) \mathrm{d} s} \\
& =C_{2} \mathrm{e}^{\int_{0}^{t}-2 \dot{\theta}(s) / \theta(s) \mathrm{d} s} \cdot \mathrm{e}^{\int_{0}^{t} a_{1}(s) \mathrm{d} s} \\
& =C_{2} \frac{1}{\theta(t)^{2}} \mathrm{e}^{\int_{0}^{t} a_{1}(s) \mathrm{d} s} \\
& u(t)=C_{1}+\int_{0}^{t} \frac{C_{2}}{\theta(\xi)^{2}} \mathrm{e}^{\int_{0}^{\xi} a_{1}(s) \mathrm{d} s} \mathrm{~d} \xi .
\end{aligned}
$$

From (20) and (21), $v(t)$ can be derived as:

$$
v(t)=C_{1}+\int_{0}^{t} \frac{C_{2}}{\theta(\xi)^{2}} \mathrm{e}^{\xi_{0}^{\xi} a_{1}(s) \mathrm{d} s} \mathrm{~d} \xi+C_{2} \frac{1}{\theta(t) \dot{\theta}(t)} \mathrm{e}_{0}^{\int_{0}^{t} a_{1}(s) \mathrm{d} s} .
$$

Therefore, the basic solution $Y(t)$ for the system (9) can be derived as:

$$
\begin{gathered}
Y(t)=\left(\begin{array}{cc}
\theta(t) & Y_{1}(t) \\
\dot{\theta}(t) & Y_{2}(t)
\end{array}\right) \\
Y_{1}(t)=\theta(t)\left(C_{1}+\int_{0}^{t} \frac{C_{2}}{\theta(\xi)^{2}} \mathrm{e}_{0}^{\int_{0}^{\xi} a_{1}(s) \mathrm{d} s} \mathrm{~d} \xi\right) \\
Y_{2}(t)=\dot{\theta}(t)\left(C_{1}+\int_{0}^{t} \frac{C_{2}}{\theta(\xi)^{2}} \mathrm{e}^{\int_{0}^{\xi} a_{1}(s) \mathrm{d} s} \mathrm{~d} \xi\right)+\frac{C_{2}}{\theta(t)} \mathrm{e}_{0}^{t} a_{1}(s) \mathrm{d} s .
\end{gathered}
$$


The period matrix is $U=Y(T) Y(0)^{-1}$ and then the eigenvalues of $U$ become Floquet multipliers. These values can be calculated directly from $Y(t), Y_{1}(t)$ and $Y_{2}(t)$ as:

$$
1, \mathrm{e}^{\int_{0}^{T} a_{1}(t) \mathrm{d} t} .
$$

These values are also the eigenvalues of the Poincare maps of the system (9).

The eigenvalue 1 in (23) corresponds to the eigenvector which is tangent to the periodic orbit. Then, the magnitude of the lest of eigenvalue has to be smaller than 1 to be the periodic motions of the system (9) orbital stable.

From the above, we can conclude that periodic motions of the system (9) become orbital stable if and only if:

$$
\begin{aligned}
& \mathrm{e}^{\int_{0}^{T} a_{1}(t) \mathrm{d} t}<1=\mathrm{e}^{0} \longleftrightarrow \\
& \int_{0}^{T} a_{1}(t) \mathrm{d} t<0 \longleftrightarrow \\
& \int_{0}^{T}\left(\frac{\partial}{\partial V}\left(\frac{1}{m} G(X) f_{\mathrm{m}}(t, X, V)\right)\right) \mathrm{d} t<0 .
\end{aligned}
$$

Remark 1. Mathematically, the above discussions are valid for any periodic motions. However, from a physiologic viewpoint, they becomes significant only when a periodic motion is feasible for the system. That is, only a periodic motion where activation function $E(t)$, which can achieve the movement that exists under the condition $0 \leqslant E(t) \leqslant 1$, is intended.

\section{Discussion}

\subsection{Physiological Meaning of the Eased Condition}

We proved that the stability condition (10) in the previous work can be eased to condition (13) by limiting the target motion to periodic motions. In particular, it is noted that the condition concerning $a_{2}$ is eliminated in our result (13). For example, from the condition concerning $a_{2}$ in (10), we get:

$$
\begin{aligned}
& \frac{1}{m}\left(\frac{\partial G(X)}{\partial X} f_{\mathrm{m}}+G(X) \frac{\partial f_{\mathrm{m}}}{\partial X}\right)<0 \longleftrightarrow \\
& \frac{\partial G(X)}{\partial X} f_{\mathrm{H}}+G(X) \frac{\partial f_{\mathrm{H}}}{\partial X}<0 \longleftrightarrow \\
& \frac{\partial f_{\mathrm{H}}}{\partial X}<-\frac{1}{G(X)} \frac{\partial G(X)}{\partial X} f_{\mathrm{H}},
\end{aligned}
$$

because $m>0, E>0$ and $G(X)>0$. The right-hand side is negative because all the terms included are positive. Therefore, the function $\partial f_{\mathrm{H}} / \partial X$ must be more negative than the right-hand side. During concentric contraction this inequality could be attainable. For eccentric contractions, the situation is completely different. If 
the velocity $v_{\mathrm{m}}$ is negative, $\partial f_{\mathrm{H}} / \partial X$ becomes positive. Therefore, this inequality is false for eccentric contractions. So, in Ref. [4], the conclusion, 'Acting eccentrically, the Hill-simple model is not stable. Acting concentrically, the Hill-simple model is stable if the contraction velocity of the muscle is fast enough' was drawn.

On the contrary, our result (13) does not seem so surprising because the class of target motion is narrowed. However, we can show that this result has quite interesting properties.

If the following inequality:

$$
\frac{\partial}{\partial V}\left(f_{\mathrm{m}}(t, X, V)\right)<0,
$$

is always satisfied, (13) is also always satisfied because $m>0$ and $G(X)>0$, which was described in Section 2. Here, the propriety of $f_{\mathrm{H}}(X, V)$ should be noted. As described in (7), the force-velocity relationship $f_{\mathrm{H}}(X, V)$ is monotonically decreasing. Therefore, (24) is always satisfied because the activation function of muscle $E(t)$ has a value from 0 to 1 , as in (13).

Consequently, the following corollary can be derived.

Corollary 1. The periodic motion of the system (8) is (locally) always stable if the motion is feasible, i.e., if activation $E(t)$, which achieves the motion, can be designed and always meets the condition $0 \leqslant E(t) \leqslant 1$.

This corollary has very strong results because, for periodic motions, the local stability of the motion can only be certified by the properties of musculoskeletal systems: even a spinal feedback loop (reflex) is not necessary. The fact that the force-velocity relationship is monotonically decreasing is one of the most popular muscle properties, which has already been claimed in previous works [4, 10], so it may have a large role in the stability of motion. However, if only periodic motions are intended, it seems quite interesting that this property plays a crucial role in the guarantee of the stability of motion.

\subsection{Variation of the Muscle Model}

Up to now, we have discussed stability with the simplest muscle model (Fig. 3a) that consists only of the contractile element. However, models in which elastic or/and damper elements are connected in parallel to the contractile element (Fig. 3b) or in which an elastic element is connected in series to the contractile element (Fig. 3c) are often used as more realistic muscle models. Thus, we discuss how stability criteria change when a model in which elastic or damper elements are connected in parallel to the contractile element is adopted, assuming that a tendon is stiff enough to disregard the series elastic elements.

In this case, the muscle force can be described as follows by the sum to originate in each of the contractile elements (CE), elastic elements (PEC) and damper 


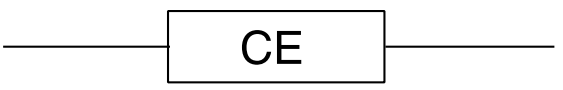

(a)

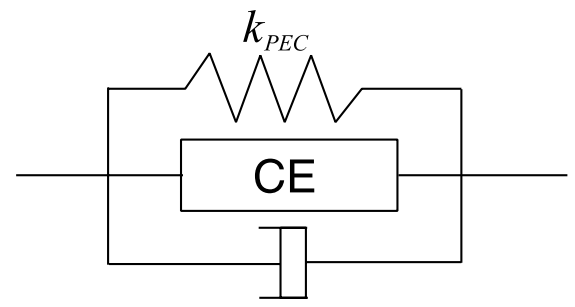

(b)

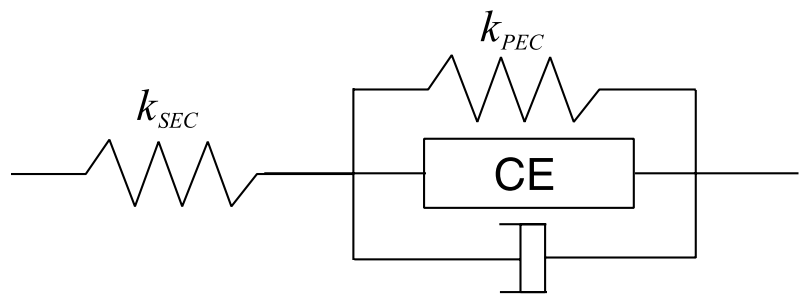

(c)

Figure 3. Variations of the muscle model. (a) Simple Hill model. (b) Hill-PEC-DC model. (c) Hill-PEC-DC-SEC model.

elements (DC):

$$
\begin{aligned}
f_{\mathrm{m}} & =E(t) f_{\mathrm{H}}(X, C)+f_{\mathrm{PEC}}+f_{\mathrm{DC}} \\
& =E(t) f_{\mathrm{H}}(X, C)+k_{\mathrm{PEC}}\left(\Delta l_{\mathrm{pec}}+\Delta l_{\mathrm{offset}}\right)-D v_{\mathrm{m}},
\end{aligned}
$$

where:

$$
\Delta l_{\mathrm{pec}}=-\int_{0}^{t} v_{\mathrm{m}}(\tau) \mathrm{d} \tau=-\int_{0}^{t} G(\tau) V(\tau) \mathrm{d} \tau,
$$

$\Delta l_{\text {offset }}$ is the difference of PEC length between the natural length of PEC and the length of PEC at $t=0, k_{\mathrm{PEC}}$ is the stiffness of the PEC, and $D$ is the damping coefficient of the DC.

When the partial derivative $f_{\mathrm{m}}(X, V)$ of $V$ is calculated while noting (3), the following inequality can be derived:

$$
\frac{\partial}{\partial V} f_{\mathrm{m}}(t, X, V)=E(t) \frac{\partial f_{\mathrm{H}}(X, V)}{\partial V}-D G(X)<0 .
$$

From this inequality, it can be said that (24) is always consistent when the model (25) is used as a muscle model and the periodic motions are always locally stable. 


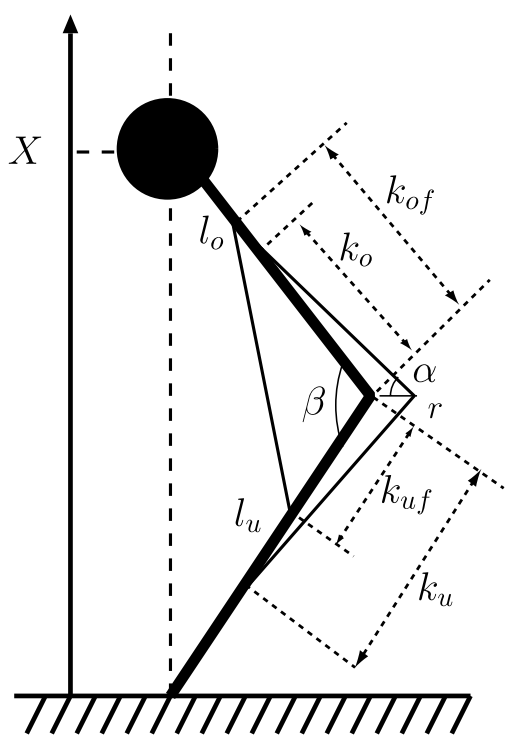

Figure 4. Geometric model of the knee joint with agonist and antagonist muscles. $l_{\mathrm{o}}, l_{\mathrm{u}}$ : length of the thigh and the shank; $k_{\mathrm{o}}, k_{\mathrm{u}}$ : length of the extensor from attachment to the middle of the patella to its insertion; $k_{\mathrm{of}}, k_{\mathrm{uf}}$ : attachments of the flexor muscle at the femur and tibia, respectively, $r$ : distance between the center of rotation and mid patella to its insertion; $m$ : body mass; $X$ : vertical displacement of the location of the hip.

\subsection{Variation of the Skeletal Model}

The linkage model with one muscle (Fig. 1) has been considered because only the flexor muscle is enough to generate a simple vertical one-dimensional movement. However, each joint of animals usually has at least two muscles, i.e., an extensor muscle and a flexor muscle. Here, we discuss how stability criteria change when the knee joint has extensor and flexor muscles (Fig. 4).

In this case, the geometric transformation between the ground reaction force $F(X, V)$ and extensor and flexor muscle force $f_{\mathrm{me}}(t, X, V)$ and $f_{\mathrm{mf}}(t, X, V)$ can be described by function $G_{\mathrm{e}}(X), G_{\mathrm{f}}(X)[5]$ :

$$
F_{\mathrm{G}}(t, X, V)=G_{\mathrm{e}}(X) \cdot f_{\mathrm{me}}(t, X, V)-G_{\mathrm{f}}(X) \cdot f_{\mathrm{mf}}(t, X, V),
$$

where:

$$
\begin{gathered}
G_{\mathrm{e}}(X)=\frac{r \sin \alpha}{l_{\mathrm{o}} l_{\mathrm{u}} \sin \beta} X \\
G_{\mathrm{f}}(X)=\frac{k_{\mathrm{of}} k_{\mathrm{uf}} \cdot X}{l_{\mathrm{o}} l_{\mathrm{u}} \sqrt{k_{\mathrm{of}}^{2}+k_{\mathrm{uf}}^{2}-\left(k_{\mathrm{of}} k_{\mathrm{uf}}\right) /\left(l_{\mathrm{o}} l_{\mathrm{u}}\right)\left(l_{\mathrm{o}}^{2}+l_{\mathrm{u}}^{2}-X^{2}\right)}} \\
\beta=2 \alpha+\arcsin \left(\frac{r}{k_{\mathrm{o}}} \sin \alpha\right)+\arcsin \left(\frac{r}{k_{\mathrm{u}}} \sin \alpha\right)
\end{gathered}
$$




$$
X=\sqrt{l_{\mathrm{o}}^{2}+l_{\mathrm{u}}^{2}-2 l_{\mathrm{u}} l_{\mathrm{o}} \cos \beta} .
$$

Note that inequalities $G_{\mathrm{e}}(X)>0$ and $G_{\mathrm{f}}(X)>0$ always also consist from the range of the movement of the knee joint $(0<\alpha<\pi$ and $0<\beta<\pi)$. With the same functions $G_{\mathrm{e}}(X)$ and $G_{\mathrm{f}}(X)$, the relationship between $V$ and $v_{\mathrm{me}}$ and $v_{\mathrm{mf}}$, i.e., extensor and flexor contractile velocity, can be written as:

$$
v_{\mathrm{me}}=G_{\mathrm{e}}(X) \cdot V \quad v_{\mathrm{mf}}=-G_{\mathrm{f}}(X) \cdot V .
$$

With the same muscle model (4)-(6):

$$
f_{\mathrm{m}\{\mathrm{e}, \mathrm{f}\}}(t, X, V)=E_{\{\mathrm{e}, \mathrm{f}\}}(t) f_{\mathrm{H}}(X, V),
$$

the dynamical equation of the system (Fig. 4) can be described as:

$$
\frac{1}{\mathrm{~d} t}\left(\begin{array}{l}
X \\
Y
\end{array}\right)=\left(\begin{array}{c}
V \\
\frac{1}{m} F_{\mathrm{G}}(t, X, V)-g
\end{array}\right)\left(\begin{array}{l}
X \\
Y
\end{array}\right) .
$$

Considering that the agonist/antagonist muscle model is different from the single muscle model only in $F_{\mathrm{G}}$, the local stability condition of this model (29) for periodic motions can be easily derived as follows (the proof of the Theorem 2 is omitted because the proof is basically the same as the proof of Theorem 1).

Theorem 2. System (29) becomes stable for periodic motions if and only if:

$$
\int_{0}^{T} b_{1}(t) \mathrm{d} t<0 \Longleftrightarrow \int_{0}^{T}\left(\frac{\partial}{\partial V}\left(\frac{1}{m} F_{\mathrm{G}}(t, X, V)\right)\right) \mathrm{d} t<0,
$$

where:

$$
b_{1}=\frac{\partial}{\partial V}\left(\frac{1}{m} F_{\mathrm{G}}(t, X, V)\right) \quad b_{2}=\frac{\partial}{\partial X}\left(\frac{1}{m} F_{\mathrm{G}}(t, X, V)\right) .
$$

Moreover, as well as the case of the model with a single muscle, the following coexist:

(i) The force-velocity relationships are always monotonically decreasing, i.e., $\partial / \partial v_{\mathrm{me}} f_{\mathrm{me}}<0$ and $\partial / \partial v_{\mathrm{mf}} f_{\mathrm{mf}}<0$.

(ii) $G_{\mathrm{e}}(X)>0$ and $G_{\mathrm{f}}(X)>0$.

(iii) $E_{\mathrm{e}}(t)>0$ and $E_{\mathrm{f}}(t)>0$.

Using the above property (i) and (27):

$$
\frac{\partial}{\partial V} f_{\mathrm{me}}<0 \quad \frac{\partial}{\partial V} f_{\mathrm{mf}}>0,
$$

can be derived.

From these facts:

$$
\frac{\partial}{\partial V}\left(F_{\mathrm{G}}(t, X, V)\right)=G_{\mathrm{e}} E_{\mathrm{e}} \frac{\partial}{\partial V} f_{\mathrm{me}}-G_{\mathrm{f}} E_{\mathrm{f}} \frac{\partial}{\partial V} f_{\mathrm{mf}}<0,
$$

can be derived as well as the case of the model with a single muscle. 
Consequently, a similar corollary as Corollary 1 is also concluded for the system (29). That is, also for the agonist/antagonist muscle model, the periodic motion of the system (31) is (locally) always stable if activation $E_{\mathrm{e}}(t)$ and $E_{\mathrm{f}}(t)$, which achieve the periodic motion, can be designed and always meet for condition $0 \leqslant E_{\mathrm{e}, \mathrm{f}}(t) \leqslant 1$.

\section{Conclusions}

In this paper, we discussed the condition of stability for movements driven by a very simple musculoskeletal system. By limiting the target motions to periodic motions and using Floquet theorem, we derived an eased stability condition compared to the previous research. In addition, considering the force-velocity relationship of muscles, which is the one of most popular properties, we showed that the eased stability condition was always satisfied, i.e., the periodic motions of the musculoskeletal system were always locally stable.

In this paper, the activation function $E(t)$ is not discussed. Of course, for various stable movements of animals, it is quite important that the activation function $E(t)$, which is an input to a muscle, is designed appropriately with feedback inputs from sensory organs. However, the results of this paper, which show that the musculoskeletal system itself has 'self-stability' especially for periodic motions to some extent, are very interesting and then it is worthwhile to clarify the generation mechanism of the stable movements of human or animals.

\section{Acknowledgements}

This work has been partially supported by a Grant-in-Aid for Scientific Research on Priority Areas 'Emergence of Adaptive Motor Function through Interaction between Body, Brain and Environment' from the Japanese Ministry of Education, Culture, Sports, Science and Technology.

\section{References}

1. G. E. Loeb, Control implications of musculoskeletal mechanics, in: Proc. 21st Annu. IEEE Int. Conf. on Engineering in Medicine and Biology, Atlanta, GA, pp. 13-16 (1999).

2. A. J. von Soest and M. F. Bobbert, The contribution of muscle properties in the control of explosive movements, Biol. Cybernet. 69, 195-204 (1993).

3. K. Gerritsen, A. van Bogert, M. Hulliger and R. Zernicke, Intrinsic muscle properties facilitate locomotor control - a computer simulation study, Motor Control 2, 206-220 (1998).

4. H. Wagner and R. Blickhan, Stabilizing function of skeletal muscles: an analytical investigation, J. Theor. Biol. 199, 163-179 (1999).

5. H. Wagner and R. Blickhan, Stabilizing function of antagonistic neuromusculoskeletal systems: an analytical investigation, Biol. Cybernet. 89, 71-79 (2003).

6. S. Grillner, Control of locomotion in bipeds, tetrapods and fish, in: Handbook of Physiology. The Nervous System. Motor Control, Vol. II, pp. 1179-1236. Bethesda, MD, American Physiological Society (1981). 
7. M. Sust, T. Schmalz and S. Linnenbecker, Relationship between distribution of muscle fibres and invariables of motion, Hum. Mov. Sci. 16, 533-546 (1997).

8. A. V. Hill, The heat of shortening and the dynamic constants of muscle, Proc. R. Soc. Lond. B Biol. Sci. 126, 136-195 (1938).

9. J. L. Van Leeuwen, Mechanics of animal locomotion, Adv. Comp. Environ. Physiol. 11, 190-250 (1992).

10. I. Brown and G. Loeb, A reductionistic approach to creating and using neuro-musculoskeletal models, in: Biomechanics and Neural Control of Posture and Movement, pp. 148-163. Springer, Berlin (2000).

\section{About the Authors}

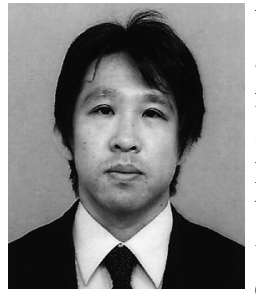

Yasuhiro Sugimoto received the BS degree in Mechanical Engineering, in 2000, and the MS and PhD degrees in Informatics, in 2002 and 2005, respectively, all from Kyoto University, Kyoto, Japan. From 2005 to 2008, he was a Research Associate in the Department of Aeronautics and Astronautics, Graduate School of Engineering, Kyoto University. In 2008, he joined Thinktube Inc. and has also been a part-time Lecturer at Department of Mechanical Engineering, Kobe University. His research interests include dynamics and control of robotic systems, especially legged robots, and analysis locomotion in humans and animals. He is a member of the RSJ, SICE and JSME.

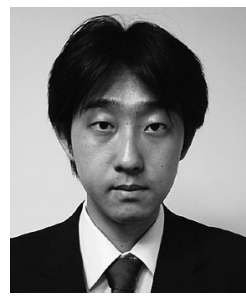

Shinya Aoi received the BE, ME and PhD degrees from the Department of Aeronautics and Astronautics, Kyoto University, Kyoto, Japan, in 2001, 2003 and 2006, respectively. From 2003 to 2006, he was a Research Fellow of the Japan Society for the Promotion of Science. From 2006 to 2007, he was a COE Assistant Professor at Kyoto University. Since 2007, has been an Assistant Professor in the Department of Aeronautics and Astronautics, Graduate School of Engineering, Kyoto University. His research interests include dynamics and control of robotic systems, especially legged robots, and analysis and simulations of locomotion in humans and animals. He is a member of the IEEE, SICE and RSJ.

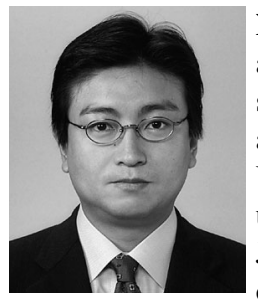

Naomichi Ogihara received the BS degree in Mechanical Engineering, in 1995, and the MS and PhD degrees in Biomedical Engineering, in 1997 and 2000, respectively, all from Keio University, Japan. He is currently an Assistant Professor at the Laboratory of Physical Anthropology, Graduate School of Science, Kyoto University. His current research interests include biomechanical analyses and simulations of locomotion in humans and non-human primates. He is a member of the Japan Society of Mechanical Engineers, Society of Biomechanisms Japan, Society of Instrument and Control Engineers, Anthropological Society of Nippon, Primate Society of Japan, International Society of Biomechanics, and American Association of Physical Anthropologists. 


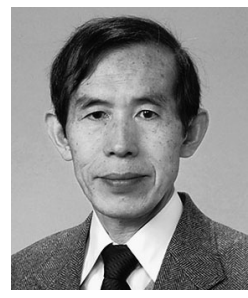

Kazuo Tsuchiya received the BS, MS and $\mathrm{PhD}$ degrees in Engineering from Kyoto University, Kyoto, Japan, in 1966, 1968, and 1975, respectively. From 1968 to 1990, he was a Research Member of the Central Research Laboratory, Mitsubishi Electric Corporation, Amagasaki, Japan. From 1990 to 1995, he was a Professor at the Department of Computer Controlled Machinery, Osaka University, Osaka, Japan. From 1995 to 2007, he was a Professor at the Department of Aeronautics and Astronautics, Kyoto University. In 2007, he retired from Kyoto University and became a Professor Emeritus of Kyoto University. He is currently a Professor in the Department of Energy and Mechanical Engineering, Faculty of Science and Engineering, Doshisha University. His fields of research include dynamic analysis, guidance, and control of space vehicles, and nonlinear system theory for distributed autonomous systems. 
Copyright of Advanced Robotics is the property of VSP International Science Publishers and its content may not be copied or emailed to multiple sites or posted to a listserv without the copyright holder's express written permission. However, users may print, download, or email articles for individual use. 
Copyright of Advanced Robotics is the property of VSP International Science Publishers and its content may not be copied or emailed to multiple sites or posted to a listserv without the copyright holder's express written permission. However, users may print, download, or email articles for individual use. 